\title{
Uma solução m-Health para apoio à educação em saúde com foco na mudança de comportamento para hábitos saudáveis
}

\author{
Maria Lúcia Kroeff Barbosa1, Mário Figueiró Zemor², Luciano Zancan², \\ Valter Roesler ${ }^{2}$, Márcia Rosa da Costa ${ }^{3}$, Sílvio César Cazella ${ }^{1,3}$ \\ ${ }^{1}$ Programa de Pós-Graduação em Informática na Educação - UFRGS \\ ${ }^{2}$ Instituto de Informática - UFRGS \\ ${ }^{3}$ Programa de Pós-Graduação em Ensino na Saúde - UFCSPA
}

malukroeff@yahoo.com.br, mario.ufrgs.inf@gmail.com, lzancan@inf.ufrgs.br, roesler@inf.ufrgs.br, marciarc.ufcspa@gmail.com, silvio.ufcspa@gmail.com

Resumo. Este artigo apresenta uma solução m-Health para apoio à educação em saúde constituida por um aplicativo para Android no dispositivo móvel cliente e uma aplicação em máquina servidora. Diferentes conceitos e teorias estão integrados visando aumentar a motivação do usuário, com foco na mudança de comportamento para hábitos mais saudáveis e melhoria da qualidade de vida. Uma avaliação da interface da aplicação foi realizada através de questionário baseado na ISO 9241-11 e ABNT ISO/IEC 25062:2011 junto a oito especialistas. Além disto, o questionário WHOQOLbref foi disponibilizado aos usuários via app, e feedbacks sobre conteúdos também são analisados e trazidos para discussão.

Palavras-Chave: m-Health, educação em saúde, comportamento, qualidade de vida

\section{An m-Health solution to support health education with a focus on behavior change for healthy habits}

\begin{abstract}
This article presents an m-Health solution to support health education consisting of an Android application on the mobile device client and an application on a server machine. Different concepts and theories are integrated to increase the motivation of the user, focusing on changing behavior to healthier habits and improving the quality of life. An evaluation of the application interface was carried out through a questionnaire based on ISO 9241-11 and ABNT ISO / IEC 25062: 2011 with eight specialists. In addition, the WHOQOL-bref questionnaire was made available to users via the app, and many feedbacks on content are also analyzed and brought to the discussion.
\end{abstract}

Keywords: m-Health, health education, behavior, quality of life

\section{Introdução}

A World Health Organization [2012] conceitua mobile-Health ou m-Health como o uso das Tecnologias da Informação e Comunicação (TICs) para cuidados com a saúde mediante a utilização de dispositivos móveis. Conforme um levantamento da 
Research2Guidance ${ }^{1}$, existem 325.000 aplicativos de saúde disponíveis nas principais lojas e os com maior potencial de sucesso para o $m$-Health estão relacionados ao tratamento de doenças crônicas como diabetes e obesidade, sendo que a América do Sul apresenta menos de 5\% deste mercado. Segundo um estudo publicado na revista The Lancet $^{2}$, o Brasil aparece como o $5^{\circ}$ país mais obeso do mundo. Barbosa et al. [2016] apresentaram um estudo com as principais funcionalidades de aplicativos móveis direcionados ao controle da obesidade, onde foi constatado que intervenções via smartphones e tablets estão sendo bem aceitas entre usuários reais. Os resultados apontaram que os aplicativos melhor avaliados são os gratuitos e que oferecem conteúdos mais completos em relação a hábitos alimentares e de atividade física. Os autores salientam que ações que visam a utilização das TICs, em prol da saúde, não podem desconsiderar o lado educacional, da Educação em Saúde.

Segundo Costa et al. [2014], o conceito de saúde 2.0 une o uso das tecnologias e ferramentas da web 2.0 à aprendizagem informal, e apontam que as tecnologias móveis favorecem a autoaprendizagem, as relações sociais, o compartilhamento de experiências e a aprendizagem colaborativa, proporcionando o acesso ao conhecimento, em qualquer lugar e a qualquer momento. A modificação comportamental, impulsionada pelas mudanças nos cuidados de saúde, centra-se numa perspectiva autorregulatória e de autocuidado que considera que as pessoas têm capacidade para regular o seu próprio comportamento, desde que possuam a motivação e as competências necessárias para tal [Marques \& Teixeira, 2014]. Sendo assim, entende-se ser relevante desenvolver e validar um aplicativo direcionado à população brasileira, onde diferentes teorias e conceitos são integrados a fim de favorecer a autoaprendizagem, procurando colocar seu usuário como agente ativo e responsável por cuidados relacionados à sua saúde.

Com base no exposto, o objetivo principal deste artigo é apresentar uma solução m-Health para apoio à educação em saúde, intitulada Emagreça@Saudável. O texto encontra-se organizado em 5 seções. A seção 2 traz a solução (sistema), bem como as teorias e diretrizes que fundamentam sua construção. A seção 3 mostra as principais interfaces do aplicativo para smartphones sensíveis ao toque, bem como para o servidor via web. A seção 4 descreve sua implementação e a seção 5 mostra os resultados das avaliações da interface do aplicativo e da qualidade de vida de alguns usuários, bem como feedbacks dos mesmos sobre conteúdos disponibilizados. Na seção 6, são apresentadas as considerações finais.

\section{Emagreça@Saudável}

A solução m-Health denominada Emagreça@Saudável é um sistema de apoio à educação em saúde que visa a melhoria da qualidade de vida do seu usuário por meio da conscientização para hábitos mais saudáveis, onde se busca salientar questões pertinentes ao sobrepeso e obesidade. As teorias comportamentais e diretrizes que embasam a construção do sistema proposto são: o Modelo Transteórico de Mudança de Comportamento (MTT) ${ }^{3}$, direcionado especificamente para a compreensão do processo de mudança de comportamentos-problema e a busca por hábitos saudáveis; o Modelo de

\footnotetext{
${ }^{1} \mathrm{http}: / /$ research2guidance.com/r2g/r2g-mHealth-App-Developer-Economics-2016.pdf

2 http://www.thelancet.com/pdfs/journals/lancet/PIIS0140-6736(16)30054-X.pdf

3 http://www.hbftpartnership.com/documents/uploadResources/TranstheoreticalT-Prochaska1982.pdf
} 
Comportamento de $\mathrm{Fogg}^{4}$; e as sete diretrizes de Margaret Morris ${ }^{5}$. Tais conceitos e teorias foram descritos em Barbosa et al. [2017].

A intenção é oportunizar a mudança de comportamento utilizando tecnologias persuasivas. Foram levados em consideração os princípios de design sugeridos por Inostroza et al. [2013], voltados para interfaces de smartphones sensíveis ao toque com foco na usabilidade, bem como a satisfação, utilidade e facilidade de uso, conforme sugere a norma Brasileira ABNT ISO/IEC 25062:2011 [2011]. Contextualmente, o Emagreça@Saudável abrange: objetivo do sistema (disponibilizar um aplicativo para dispositivos móveis que envolva cuidados com a saúde); características do usuário (características gerais das pessoas que vão utilizar); e características do dispositivo (qualidades e limitações de uso de um smartphone). O sistema foi refinado do último estudo [Barbosa et al., 2017] e está composto por: aplicativo para dispositivos móveis (interface principal por onde o usuário cadastra seus dados, executa as tarefas propostas, acompanha sua evolução e recebe orientações); redes sociais (comunidade no Facebook, canal no YouTube e WhatsApp usada como apoio aos usuários do aplicativo, com repositório de vídeos, áudios e textos com dicas de saúde, esclarecimentos sobre estilo de vida e sugestões de aulas diferenciadas); servidor (possui o banco de dados que armazena todas as informações dos usuários, além de auxiliar na comunicação com os dispositivos móveis e com as redes sociais).

\section{A Interface Implementada}

A interface implementada para o aplicativo em dispositivos móveis está baseada nas teorias usadas para a criação da interface com o usuário citadas anteriormente. Após a instalação do aplicativo no smartphone, aparece uma mensagem de boas-vindas (Figura 1a) e a necessidade de fazer o login (ou signup), onde o usuário pode escolher usar seus dados via Facebook (Figura 1b) e não precisa fazer seu cadastro pelo $a p p$.

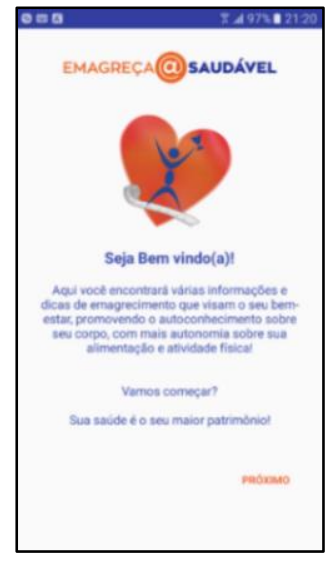

(a)

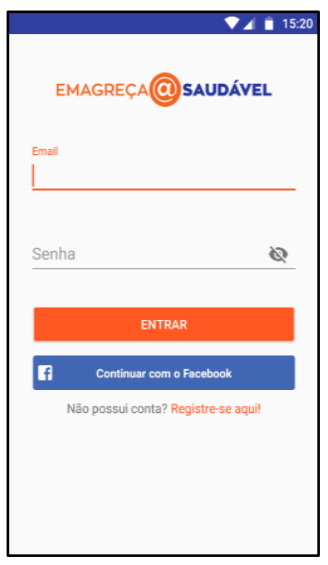

(b)

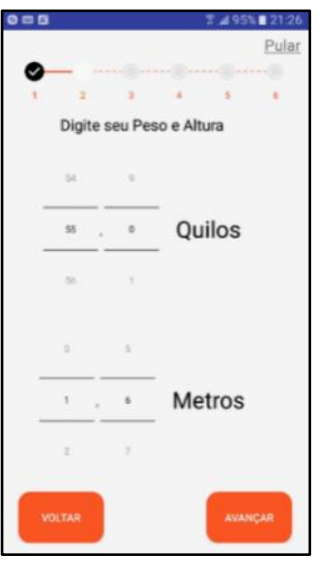

(c)

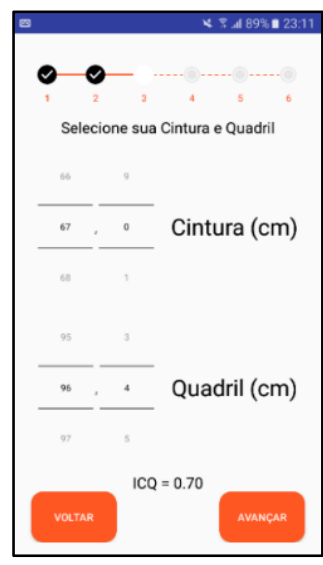

(d)

Figura 1: (a) Interface de boas-vindas; (b) Interface de entrada para o app; (c) Interface para medidas de Peso e Altura; (d) Interface para medidas de Cintura e Quadril

A Figura 2(a) apresenta a mensagem de início da $1^{\text {a }}$ etapa (Onde Tudo Começa), procurando orientar o caminho neste primeiro momento. A Figura 2(b) mostra a aba "Tarefas", com um resumo da evolução do usuário ao longo do seu processo, ou seja, quantos passos o mesmo já realizou, quanto é a meta a ser cumprida nesta etapa e

\footnotetext{
${ }^{4}$ http://www.behaviormodel.org/

${ }^{5}$ http://pt.scribd.com/doc/92691136/Motivating-Change-With-Mobile-Seven-Guidelines-2012
} 
quantos dias faltam para finalizar. As Figuras 2(b) e 2(c) apresentam opções de escolha de Atividades e Desafios e, quando as mesmas são concluídas (usuário marca o checkbox à direita), elas descem para o final da lista. As que ainda estão por fazer aparecem no topo da lista.

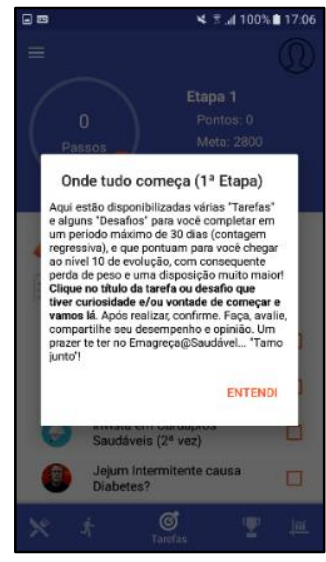

(a)

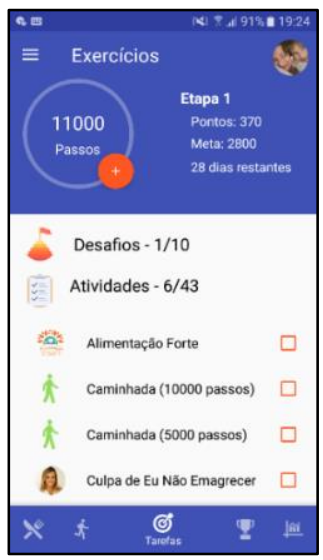

(b)

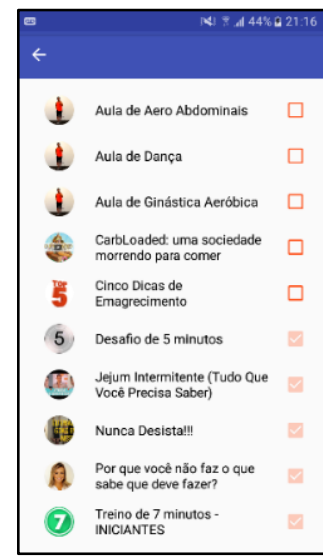

(c)

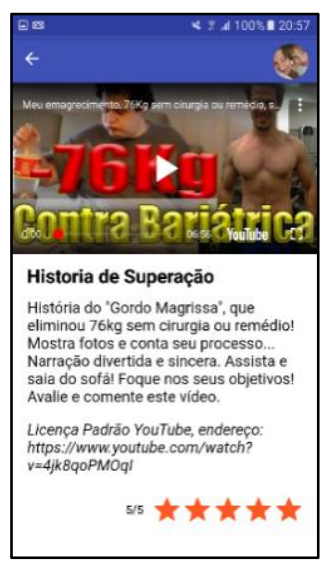

(d)

Figura 2: (a) Mensagem 1aㅡ etapa; (b) Aba de Tarefas; (c) Tela de Desafios; (d) Atividade educacional - vídeo de superação

As Atividades e Desafios (na aba de Tarefas) foram pensados para que o usuário não somente realize as tarefas solicitadas, mas entenda o motivo de estar fazendo a maioria delas através de vídeos educacionais. Desta forma, entende-se que os conteúdos podem proporcionar acesso ao conhecimento, trazendo a compreensão sobre tantas informações que se lê, vê e ouve nas mídias. Isto é, possuem objetivos educacionais que podem ser vistos, por exemplo, na Figura 2(c) em "CarbLoaded: uma sociedade morrendo para comer", Cinco Dicas de Emagrecimento" e "Jejum Intermitente (Tudo Que Você Precisa Saber)", entre outras sugestões que possibilitam ao usuário conhecer histórias de superação, dentro de um contexto parecido, inclusive, com o seu (Figura 2d). A interface possui uma barra inferior azul (Figura 2b) que permite o acesso rápido aos principais elementos da interface:

- $\rtimes$ Alimentação: informações sobre alimentos, sugestões de cardápios e receitas, dicas com base no Guia Alimentar da População Brasileira, bem como conteúdos sobre o que está "em voga", procurando desmistificar muita notícia sem comprovação científica. Ver Figura 3(a).

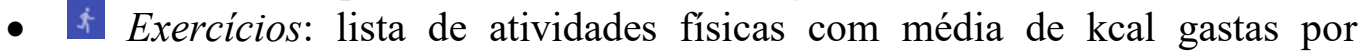
minuto. Tem opção de cadastro pelo usuário do tempo que realizou cada exercício para que contabilize em sua pontuação. Ver Figura 3(b).

- $\quad$ Pontuação: exibe a pontuação do usuário e o nível em que se encontra, buscando motivar o mesmo numa espécie de gamificação, onde é possível visualizar a pontuação dos Top 10, bem como os 3 acima e abaixo, além de "seguir" alguém que deseje.

- Evolução: exibe gráficos de evolução de peso, IMC e ICQ do usuário, visando mostrar se houve ou não melhoria nas medidas e índices.

$\mathrm{Na}$ barra superior canto esquerdo da interface aparece o ícone $\equiv$ (Figuras 3a e 3b). Clicando-se nele, é possível o acesso para: Perfil (usuário pode editar suas informações pessoais, foto, visualizar suas medidas e ver as tabelas de IMC e ICQ, conforme Figura 3d); Motivacional (mensagens motivacionais são enviadas uma vez ao 
dia e ficam armazenadas neste item); Grupo WhatsApp (finalidade de integrar o usuário em redes sociais para o mesmo receba e troque informações sobre conteúdos, além de mensagens com outras pessoas em situação similar); Configurações (permite ajustar algumas configurações, como horários das mensagens motivacionais e lembrete de medições semanais); e Perguntas Frequentes (acesso às perguntas mais frequentes sobre uso do aplicativo, bem como canais de atendimento disponibilizados via email e WhastApp). A Figura 3(c) traz a interface desta tela.

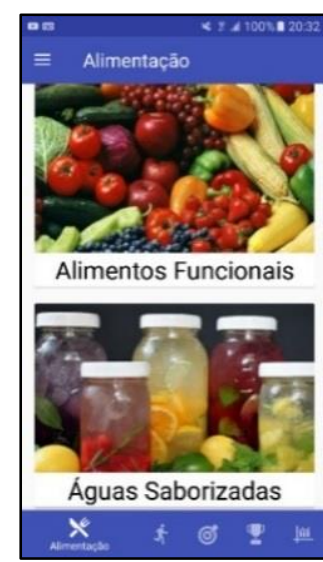

(a)

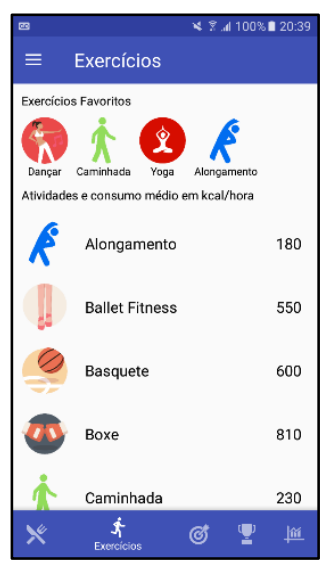

(b)

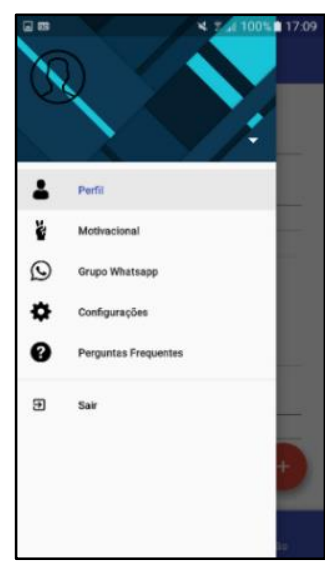

(c)

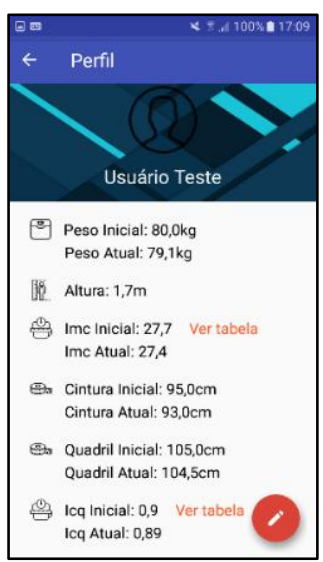

(d)

Figura 3: (a) Alimentação; (b) Exercícios; (c) Interface ícone 三; (d) Perfil

Já a interface implementada para o servidor foi idealizada com a possibilidade de edição para uma equipe multidisciplinar sem maiores conhecimentos em programação. Quando inserido e salvo qualquer material, o mesmo é automaticamente atualizado e baixado pelo aplicativo. Uma das principais telas é a de "Cadastro de Conteúdo", onde pode-se escolher entre as categorias sugeridas (Figura 4).

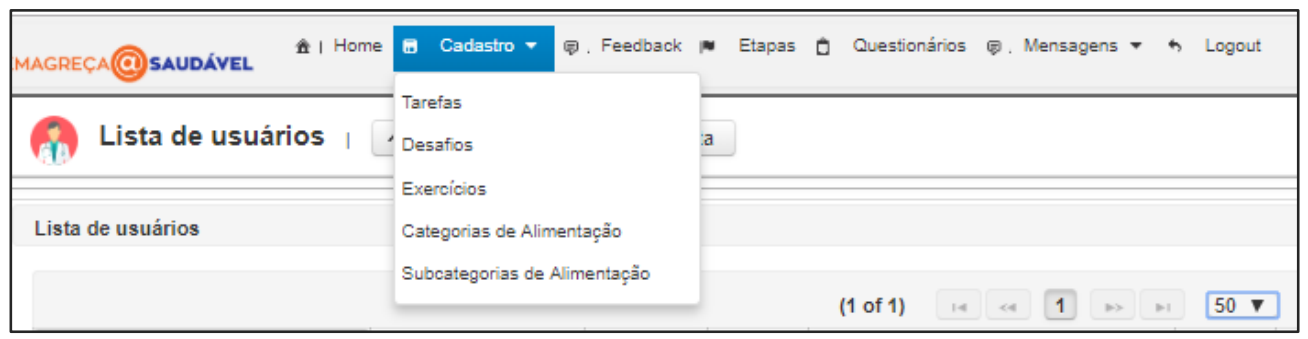

Figura 4: Cadastro de Conteúdos

A Figura 5 apresenta uma tela parcial da lista de tarefas cadastradas no sistema. Cada tarefa pode ser visualizada, editada ou excluída, bem como a cada uma é atribuído um valor de pontos (terceira coluna). Na quarta coluna, aparece a média da votação dos usuários, de 1 a 5 estrelas, sendo que as tarefas que estão com " 0.0 " não foram votadas ainda por nenhum usuário.

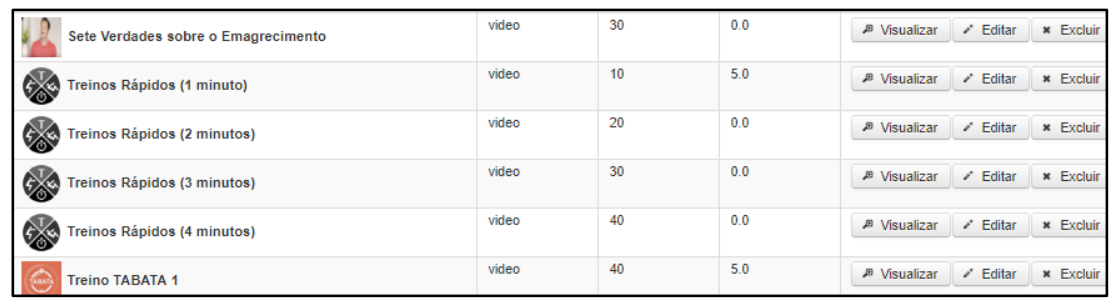

Figura 5: Lista de Tarefas cadastradas 
A lista de usuários permite visualizar rapidamente o perfil de cada participante (sexo, peso, IMC, ICQ), bem como sua pontuação e ranking (colocação) entre todos os usuários. Ingressando em um usuário específico, é possível conhecê-lo melhor, saber o tipo de exercício que ele gosta mais, tarefas que realizou, dias na semana preferidos para se exercitar, bem como verificar graficamente a tendência de perda de peso, de medidas e índices.

\section{Implementação do Sistema}

Para a implementação do aplicativo no dispositivo móvel foi decidido focar primeiramente na plataforma Android, uma vez que este é o sistema operacional móvel mais usado no mundo e no Brasil ${ }^{6}$. Usaram-se ferramentas de desenvolvimento de código nativo à plataforma pelo melhor desempenho e experiência de usuário proporcionada. Para o desenvolvimento da aplicação server-side (no servidor), foi utilizada a linguagem JAVA e empregue a arquitetura de software MVC (Model View Controller) fazendo uso do framework Java Server Faces (JSF) ${ }^{7}$. A hospedagem da aplicação foi feita com o Oracle GlassFish Server. O servidor permite o acesso a informações através de serviços REST (Representational State Transfer), gerando uma camada de interface de comunicação de dados que, por sua vez, serão disponibilizados para o aplicativo móvel, permitindo realizar as operações de CRUD (Create, Read, Update e Delete) nos dados armazenados no banco de dados. Para o gerenciamento do banco foi utilizado o PostgreSQL $L^{8}$.

Para comunicação entre aplicativo e servidor, utilizou-se o cliente HTTP Retrofit $^{9}$, que simplifica a tarefa de consumir Web Services. O cliente interessado em recuperar informações deve realizar uma requisição HTTP utilizando o método GET, POST, PUT ou DELETE. Os dados solicitados são retornados formatados no padrão $\mathrm{JSON}^{10}$ (JavaScript Object Notation). Foram criados mais de 15 Web Services, onde cada um possui pelo menos um dos quatro métodos acima. O banco de dados local usado no dispositivo móvel foi o Realm ${ }^{11}$. Além da comunicação HTTP por meio de JSONs, também foi utilizado a biblioteca OkHttp $^{12}$ e a biblioteca Picasso para, respectivamente, fazer cache e download de imagens do servidor, para serem exibidas em seus respectivos locais no aplicativo. Foi aplicado o padrão de criação Singleton ${ }^{13}$ para recuperar uma instância do banco de dados sempre que necessário, fechando tal instância quando não for mais utilizada. A estrutura de Job Queue foi definida para permitir que a camada de comunicação realize todas as trocas de mensagens em plano de fundo para não causar perda de desempenho na thread de interface de usuário. São efetuados controles, como o de conexão ativa, que só realiza uma operação de comunicação com o servidor caso a conexão esteja ativa. Caso contrário, o job entra em uma fila (job queue), para ser processado assim que a conexão com a internet estiver ativa. Para evitar manipulações de funções de várias camadas na interface de usuário, foi utilizado o padrão estrutural Façade ${ }^{14}$, no qual, com apenas uma chamada de função,

\footnotetext{
${ }^{6} \mathrm{http}: / /$ gs.statcounter.com/os-market-share/mobile/brazil

${ }^{7} \mathrm{http}: / /$ www.javaserverfaces.org/

${ }^{8}$ https://www.postgresql.org/

${ }_{9} \mathrm{https} / / /$ github.com/square/retrofit

${ }^{10}$ https://www.json.org/

$11 \mathrm{https} / / /$ realm.io/products/realm-database/

12 https://github.com/square/okhttp

${ }^{13} \mathrm{https} / / /$ en.wikipedia.org/wiki/Singleton_pattern

${ }^{14}$ https://pt.wikipedia.org/wiki/Façade
} 
como "downloadProfile()", é possível baixar o perfil do usuário do servidor e salvá-lo no banco de dados Realm sem manipulação na interface de usuário, otimizando o sistema. O aplicativo também efetua contagem de passos, que foi desenvolvido utilizando-se o próprio SDK do Android. Visando facilitar a manutenção e melhorar o desempenho, foram utilizados alguns padrões extras, como a injeção de dependências e inversão de controle. Com o framework Dagger ${ }^{15}$, foi possível obter estas propriedades, evitando que classes criem os objetos que necessitam, desacoplando-as.

\section{Avaliações e Resultados}

A fim de aperfeiçoar o sistema como um todo e entender melhor seus usuários, foram realizadas a avaliação da interface do aplicativo, a avaliação subjetiva da qualidade de vida dos usuários, bem como a avaliação de conteúdos disponibilizados no app. A avaliação da interface do app foi elaborada com base nos requisitos de ergonomia fundamentados pela ISO 9241-11 ${ }^{16}$. Um total de 35 questões buscou atender as 13 heurísticas a serem avaliadas, bem como satisfação, utilidade e facilidade de uso, conforme sugere a norma Brasileira ABNT ISO/IEC 25062:2011 [NBRISO/IEC25062, 2011]. Utilizou-se uma escala de 1 a 5 (onde 1=Discordo Totalmente, 2=Discordo Parcialmente, 3=Indiferente, 4=Concordo Parcialmente e 5=Concordo Totalmente). Além disso, caso o avaliador encontrasse problemas ou bugs, sugestões de correções, e a prioridade de realização das mesmas, foram solicitadas. Um total de 8 avaliadores especialistas (4 da área da Ciência da Computação, 2 da área da Informática na Educação, 1 da área da Psicologia e 1 da área da Saúde) usaram o app nos seus próprios smartphones. O período de avaliação foi de 20 de abril a 17 de maio de 2018 , sendo 8 avaliadores suficientes para obter resultado significativo [NBRISO/IEC25062, 2011]. A Figura 6 apresenta o gráfico com a média dos resultados.

Como se visualiza, a grande maioria fica entre 4 e 5 em relação à contemplação de todos os aspectos avaliados. Na "Personalização" (saber se o aplicativo mostrava opções de configurações a fim do próprio usuário definir algumas possibilidades), foi onde dois dos avaliadores sentiram dificuldade em localizar onde encontrar esta opção. No mais, houveram poucas dificuldades de uso, que já foram melhoradas na nova versão do aplicativo.

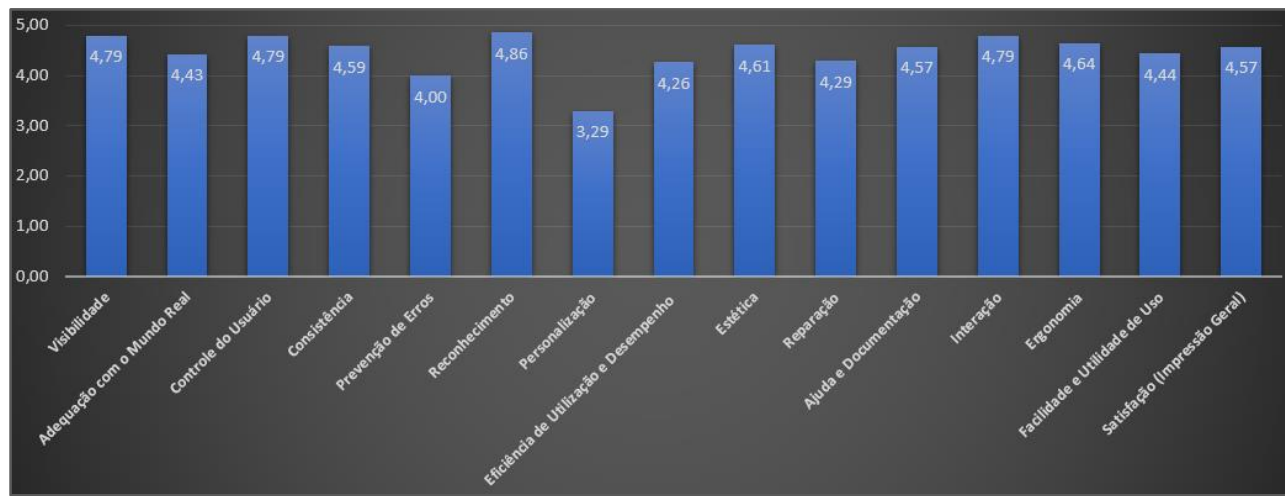

Figura 6: Resultados da avaliação da Interface do Aplicativo

\footnotetext{
${ }^{15}$ https://github.com/google/dagger

${ }^{16}$ https://www.iso.org/obp/ui/\#iso:std:iso:9241:-11:ed-2:v1:en
} 
A avaliação subjetiva da qualidade de vida é verificada pelo questionário WHOQOL-bref ${ }^{17}$, com 26 questões que medem os seguintes domínios amplos: saúde física, saúde psicológica, relações sociais e meio ambiente. Importante salientar que os instrumentos WHOQOL tem uso liberado para pesquisa acadêmica por parte da Organização Mundial da Saúde que detém o copyright destes instrumentos. Foram desenvolvidos em colaboração com vários centros em todo o mundo e amplamente testados em campo. Para aplicação com os usuários do Emagreça@Saudável, foi estruturado na plataforma GoogleDocs e seu preenchimento, para fins de pesquisa, solicitado via app. Caso o usuário deixe para preencher e enviar após seu primeiro login, sempre aparecerá uma notificação lembrando a realização desta tarefa. O período de avaliação para fins de escrita deste artigo foram os enviados até 27 de outubro de 2018, totalizando 23 respostas.

A Figura 7 apresenta alguns gráficos das respostas, principalmente em relação a como os usuários avaliam sua qualidade de vida, sua saúde, aparência física e sentimentos negativos.

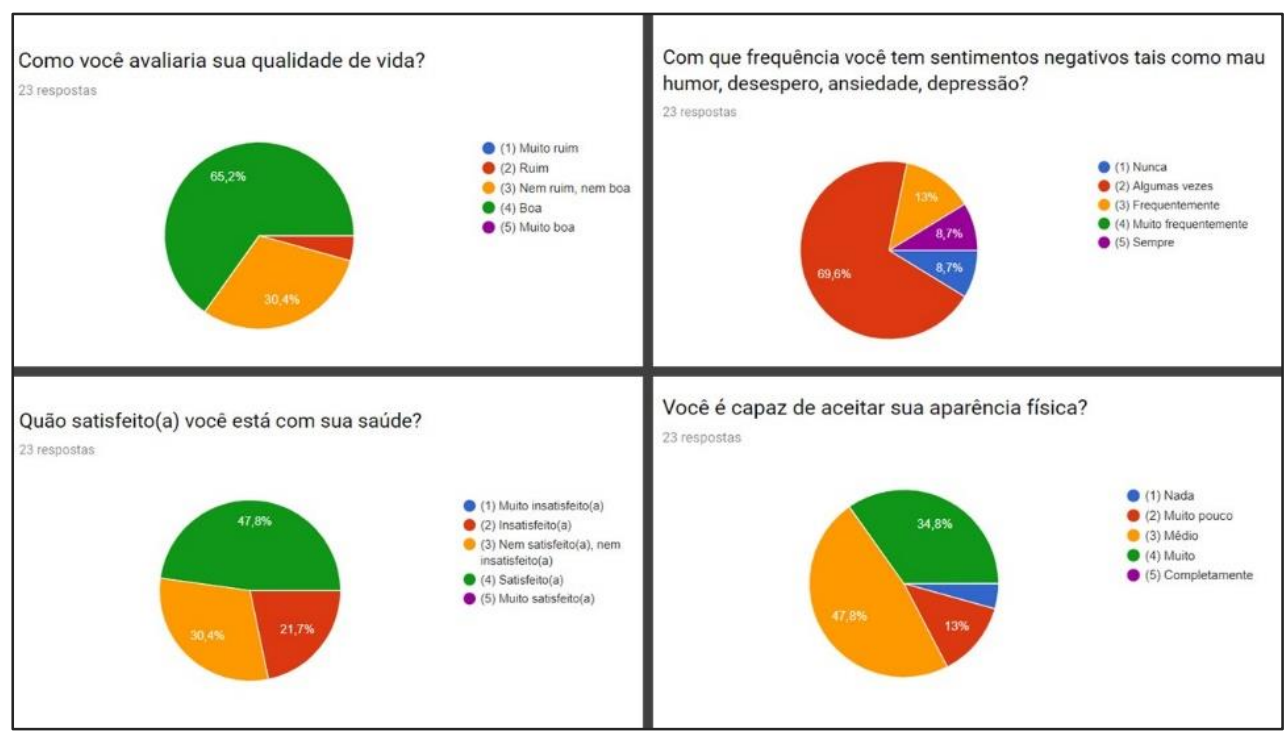

Figura 7: Resultados dos gráficos das respostas do WHOQOL-bref

No que se refere à qualidade de vida, saúde e aparência física nenhuma das respostas mostrou total satisfação. Para qualidade de vida, 15 respondentes $(66,2 \%)$ a classificam como "Boa" e $7(30,4 \%)$ como "Nem ruim, nem boa". Já na questão que trata da aparência física, $11(47,8 \%)$ responderam que são capazes de aceitar "Médio" e 3 (13\%) "Muito Pouco", sendo que 1 respondente não a aceita. Na pergunta sobre sentimentos negativos, a maioria (16 respondentes) afirma que os sente algumas vezes, sendo que 2 as sentem sempre e 2 nunca sentiram. Importante salientar que a maioria das perguntas deveriam ser respondidas com base nas últimas duas semanas. Os feedbacks, no que dizem respeito às atividades e desafios propostos, bem como às mensagens motivacionais, podem ser remetidos via app tanto por "estrelas" (1 a 5 ), como por comentário (Figura 8).

${ }^{17}$ http://www.who.int/substance abuse/research tools/whoqolbref/en/ 

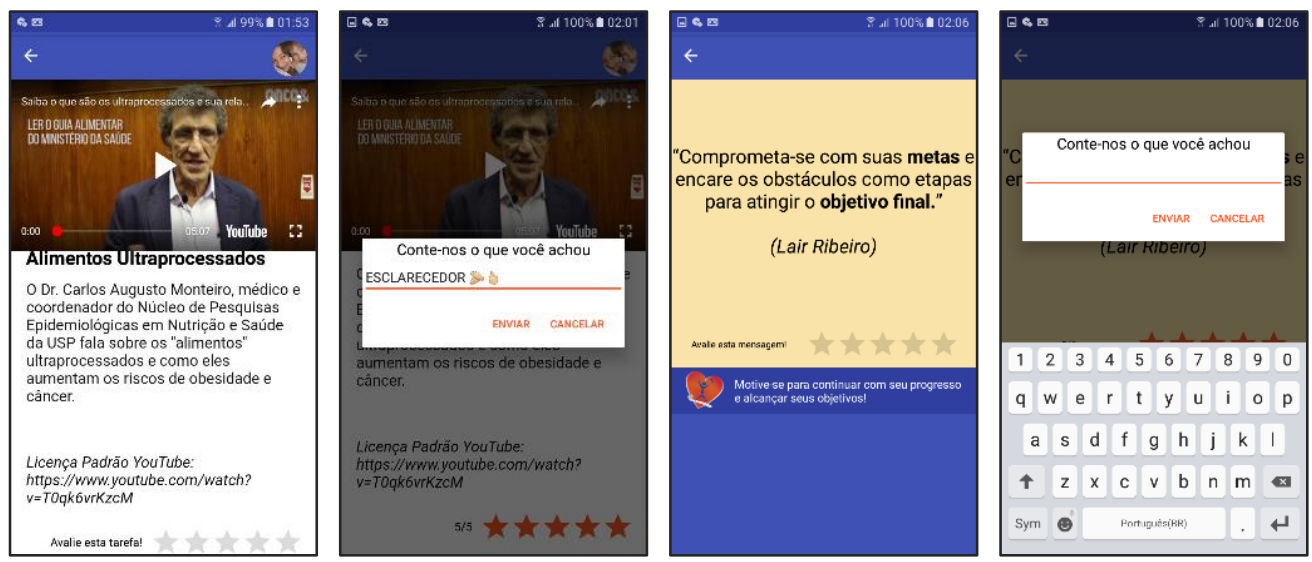

Figura 8: (a) Exemplo de atividade no app; (b) Exemplo de feedback realizado;

(c) Exemplo de mensagem motivacional; (d) Exemplo de inserção de comentário

Um total de 98 respostas foram enviadas num prazo de 46 dias, sendo 32 sobre as mensagens motivacionais, como por exemplo, a mensagem " $A$ vida não exige que sejamos os melhores, apenas que dediquemos o melhor de nossos esforços para isso", teve sua média de estrelas em 4.5 e o seguinte comentário: "Eu sempre exigi demais de mim. Sou perfeccionista e por isso me frustro demais".

Muitas atividades e desafios foram avaliados (66 comentários), e pode-se destacar alguns com pontuação média de 5 estrelas, cujo objetivo era o acesso ao conhecimento e facilitar o processo de autoaprendizagem e modificação comportamental: "Alimentação Forte", "Obesidade (Dr. João Mano José Jr.), "Sete Verdades sobre o Emagrecimento", "Só um pedacinho...", "Nunca Desista!!!". Respectivamente, trouxeram os comentários: "Gostei. Curiosamente, eu e minha esposa estamos adotando alimentos mais saudáveis na nossa dieta, com o objetivo de evitar alimentos industrializados e embutidos em geral. A informação da alimentação forte certamente vai acrescentar qualidade no nosso cardápio"; "Achei incrível que em 2012 já existisse esse conhecimento sobre os vilões serem carboidratos e conservantes, e eu só tenha recebido essas informações a partir de 2017..."; "Adorei. Percebi que tenho muitos sabotadores na família e no círculo de amizades, infelizmente"; "Show, nunca tinha pensado por esse lado”; "Nossa!!! Chorei horrores, muito lindo! Só mostra que nosso corpo é capaz de tudo, e na maioria das vezes, é a nossa mente que nos puxa pra trás...". Uma atividade foi avaliada com 1 estrela por um dos usuários. O "Dia de Alimentação Forte" remete a algumas opções de cardápio onde todos apresentam carne de gado, frango e peixe. Trouxe o seguinte comentário: "sou vegetariana... Não tem opções?”.

\section{Considerações finais}

O m-Health está em grande expansão e os aplicativos para saúde possuem grande potencial. Com as ferramentas necessárias para promoção da saúde e bem-estar, o sistema Emagreça@Saudável está embasado em teorias, modelos e diretrizes que possuem caráter persuasivo e visa auxiliar seus usuários não só na mudança, mas na permanência de um comportamento saudável. A implementação do aplicativo passou pela fase de Alfa-Teste, onde especialistas analisaram sua usabilidade com base em heurísticas de design voltadas para interfaces de smartphones sensíveis ao toque, bem como avaliações prévias para melhor conhecimento do público alvo, a fim de selecionar e direcionar adequadamente os conteúdos propostos. Pequenas correções estão sendo 
efetuadas no sistema para iniciar o Beta-teste com pacientes obesos e com sobrepeso. Os conteúdos até então disponibilizados e a forma como os mesmos são apresentados contemplou a grande maioria dos usuários do sistema, com base nos comentários remetidos. O app já se encontra disponível na Google Play para ser utilizado e receber feedbacks que auxiliem em sua melhoria. Basta digitar na ferramenta de busca da loja "Emagreça@Saudável”, selecionar, instalar e usar.

Esta pesquisa permite ainda diversos desdobramentos, como a motivação dos usuários através da gamificação e o incentivo por meio das redes sociais. Além disso, pela quantidade potencial de dados dos participantes, vários estudos de Data Mining para Big Data podem ser efetuados com as informações obtidas pelo sistema. A ideia é manter o sistema livre, de código aberto, com política de uso de dados de forma anônima, mas abertos para novas pesquisas.

\section{REFERENCIAS}

BARBOSA, M. L. K. et al. Aplicativos móveis para controle da obesidade e modelagem do emagreça@saudável. "RENOTE”. Revista Novas Tecnologias na Educação, v. 14, p. 1-10. Disponível em: http://seer.ufrgs.br/renote/article/view/67371. Acesso em setembro de 2018.

BARBOSA, M. L. K. et al. Educação permanente através de um sistema m-Health voltado ao controle da obesidade em adultos. "RENOTE". Revista Novas Tecnologias da Educação, v. 15, p. 1-10. Disponível em: $<$ http://seer.ufrgs.br/renote/article/view/75113/42553>. Acesso em setembro de 2018.

COSTA, A. A. L. et al. Recomendação Personalizada de Conteúdo para Suporte à Aprendizagem Informal no Contexto da Saúde. "RENOTE". V. 12 N 1, julho, 2014. Disponível em: <http://seer.ufrgs.br/index.php/renote/article/view/49840/31197>. Acesso em abril de 2017.

INOSTROZA, R. et al. "Usability heuristics for touchscreen-based mobile devices: Update". Disponível em: <http://jcc2013.inf.uct.cl/wpcontent/proceedings/ChileCHI/Usability\%20Heuristics\%20for\%20Touchscreenbased\%20Mobile\%20Devices\%20Update.pdf>. Acesso em janeiro de 2017.

MARQUES, M. M.; TEIXEIRA, P. J. Modificação Comportamental na Gestão do Peso: da teoria à prática. Revista Fatores de Risco. No 34. Out-Dez 2014. p. 55-66.

Disponível em:

$<$ https://www.researchgate.net/profile/Marta_Marques2/publication/271645671_Mo

dificacao_Comportamental_na_Gestao_do_Peso_da_Teoria_a_Pratica/links/54ce865 e0cf29ca810fc6fd4.pdf $>$. Acesso em maio de 2017.

NBRISO/IEC25062: Engenharia de software - Requisitos e avaliação da qualidade de produto de software (SQuaRE). 2011. Disponível em: $<$ https://www.target.com.br/produtos/normas-tecnicas/42260/nbriso-iec25062engenharia-de-software-requisitos-e-avaliacao-da-qualidade-de-produto-de-softwaresquare-formato-comum-da-industria-fci-para-relatorios-de-teste-de-usabilidade $>$. Acesso em maio de 2018.

WHO (World Health Organization). “eHealth”. Genebra: 2012. Disponível em: $<$ http://www.who.int/ehealth/en/>. Acesso em agosto de 2016. 